SANTOS, A.L.Q. et al. Anestesia de tigre d'água brasileiro Trachemys dorbigni (DUMÉRIL e BIBRON, 1835) - Testudine: Emydidae com a associação de midazolam e propofol. PUBVET, Londrina, V. 5, N. 17, Ed. 164, Art. 1109, 2011.

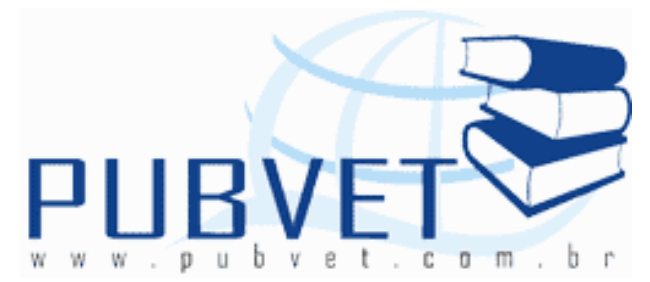

PUBVET, Publicações em Medicina Veterinária e Zootecnia.

\title{
Anestesia de tigre d'água brasileiro Trachemys dorbigni (DUMÉRIL e BIBRON, 1835) - Testudine: Emydidae com a associação de midazolam e propofol
}

André Luiz Quagliatto Santos ${ }^{1}$, Dayane Olimpia Gomes ${ }^{3}$, Cirilo Antônio de Paula Lima $^{1}$, Liliane Rangel Nascimento ${ }^{2}$, Lorena Tannús Menezes ${ }^{2}$, Árthur Paulino Sanzo Kaminishi ${ }^{2}$.

Laboratório de Ensino e Pesquisa em Animais Silvestres - LAPAS, FAMEV/UFU, e-mail: quagliatto@famev.ufu.br 1. Docente. 2. Mestrandos. 3. Médica veterinária residente.

\section{Resumo}

Os cágados apresentam fisiologia e morfologia únicas, que se diferenciam em muitos aspectos dos mamíferos. Por isso, a monitoração do paciente durante um processo anestésico ou sedativo deve ser realizada. Foram utilizados dez exemplares de Trachemys dorbigni, provenientes do rio Uberabinha, no município de Uberlândia (MG), os quais foram anestesiados com o protocolo midazolan $2 \mathrm{mg} / \mathrm{kg}$ IM e propofol $10 \mathrm{mg} / \mathrm{Kg}$ IM. Os batimentos cardíacos dos exemplares foram monitorados com o aparelho Doppler Vascular Eletrônico e durante o período trans anestésico os cágados foram observados em relação aos parâmetros estipulados. O propofol $(10 \mathrm{mg} / \mathrm{kg}$ IM) se mostrou um anestésico eficiente, promovendo relaxamento muscular e facilidade de 
SANTOS, A.L.Q. et al. Anestesia de tigre d'água brasileiro Trachemys dorbigni (DUMÉRIL e BIBRON, 1835) - Testudine: Emydidae com a associação de midazolam e propofol. PUBVET, Londrina, V. 5, N. 17, Ed. 164, Art. 1109, 2011.

manipulação do animal. Não houve significante diminuição da frequência cardíaca, porém foi observado apnéia nos quelônios anestesiados.

Palavras-chave: propofol, midazolam, Trachemys dorbigni

\section{Anesthesia of turtle Trachemys dorbigni (DUMÉRIL E BIBRON, 1835) - Testudine: Emydidae with the combination of midazolam and propofol}

\section{Summary}

The tortoises have unique physiology and morphology, which differ in many aspects of mammals. Therefore, monitoring the patient during a sedative or anesthetic procedure should be performed. We used ten copies of Trachemys dorbigni, from Uberabinha River, in Uberlândia (MG), which were anesthetized with midazolam protocol $2 \mathrm{mg} / \mathrm{kg}$ IM and propofol $10 \mathrm{mg} / \mathrm{kg}$ IM. The heartbeats of the specimens were monitored with the Electronic and Vascular Doppler device during trans anestesic, the turtles were observed for those parameters stipulated. The propofol $(10 \mathrm{mg} / \mathrm{kg}$ IM) proved an effective anesthetic, promoting muscle relaxation and ease of manipulation animal. There was no significant decrease in heart rate but it was observed apnea in the anesthetized turtle.

Keywords: propofol, midazolam, Trachemys dorbigni

\section{INTRODUÇÃO}

$\mathrm{Na}$ evolução dos vertebrados, os répteis representam um grupo transitório entre peixes, aves e mamíferos terrestres (ERNEST; BARBOUR, 1989). A classe Reptilia sofreu perda de espécies, pois das 16 ordens existentes no passado apenas três sobreviveram, dentre elas a Testudines formada por cágados, tartarugas e jabutis (ORR, 1970). O cágado tigre d'água brasileiro (Trachemys dorbigni) foi descrito pela primeira vez por Durméril e Bibrom, 1835 (FERREIRA et al,1993), é uma espécie natural do rio Guaíba no estado do Rio Grande do Sul e habita principalmente a Lagoa dos patos e o 
SANTOS, A.L.Q. et al. Anestesia de tigre d'água brasileiro Trachemys dorbigni (DUMÉRIL e BIBRON, 1835) - Testudine: Emydidae com a associação de midazolam e propofol. PUBVET, Londrina, V. 5, N. 17, Ed. 164, Art. 1109, 2011.

Banhado do Taim. Estes quelônios apresentam o corpo inteiramente coberto pelo casco (carapaça superior) e o plastrão na porção inferior, de onde somente a cabeça, os membros e a cauda emergem.

Os répteis apresentam como particularidade anatomo-fisiológica a presença do sistema porta renal, o qual coleta o sangue proveniente da cauda e região pélvica e o conduz para os rins. Tal fato explica a importância de se utilizar os membros torácicos como locais de administração de fármacos, pois, caso contrário, a droga não alcança a circulação sistêmica, sendo depurada nos rins e excretada sem exercer efeito (HOLZ et al., 1997a; HOLZ et al., 1997b). Outra peculiaridade desses animais é a presença de uma taxa metabólica extremamente baixa, que resulta em lenta metabolização e excreção de fármacos, de modo que protocolos anestésicos injetáveis provocam uma recuperação de longa duração.

Com o transporte ilegal da espécie Trachemys scripta elegans (tigre d'água americano), oriunda dos Estados Unidos, para o Brasil, surgiram vários problemas para a espécie nacional (Trachemys dorbigni), provocando desequilíbrio ambiental. Para oferecer uma alternativa ao mercado de animais de estimação e para garantir a conservação da espécie brasileira, o IBAMA (Instituto Brasileiro do Meio Ambiente e dos Recursos Renováveis) autorizou a abertura de um criadouro comercial para espécie brasileira, onde a criação é totalmente controlada por biólogos especialistas em quelônios e aberto para pesquisa e comercialização. Com a comercialização desses animais como animais domésticos, acidentes que levam a trauma tanto nos membros como no casco são comuns em ambiente domiciliar, além de doenças metabólicas que podem causar alterações fisiológicas sendo necessário a contenção farmacológica do animal para a coleta de material para exames ou para um pequeno procedimento cirúrgico.

Os veterinários que cuidam de répteis deparam-se freqüentemente com situações que requerem o uso da anestesia farmacológica para contenção desses animais. Em quelônios que se escondem em suas carapaças ou ainda animais grandes e de difícil manejo, como os jacarés, até mesmo para exame 
SANTOS, A.L.Q. et al. Anestesia de tigre d'água brasileiro Trachemys dorbigni (DUMÉRIL e BIBRON, 1835) - Testudine: Emydidae com a associação de midazolam e propofol. PUBVET, Londrina, V. 5, N. 17, Ed. 164, Art. 1109, 2011.

físico ou avaliação, requerem sedação. Em situações em que é necessária uma cirurgia para abertura do casco, como extração de corpo estranho do esôfago retirada de oviduto com ovos retidos, amputação do pênis no caso de parafimose, é preciso conhecer o protocolo para anestesiar com segurança, esses répteis (CARVALHO, 2005).

Grande quantidade de tartarugas acidentalmente engolem anzóis de pescadores, tendo que ser submetidas a esofagotomia exploratória (SANTANA, 2003). Estes são apenas alguns exemplos de casos não cirúrgico e cirúrgico que necessitam de conhecimentos em anestesiologia. Contudo existem poucas informações a esse respeito para répteis e em especial para os Testudines.

O midazolam é agente hipnótico e miorrelaxante, depressor do sistema nervoso central e derivado de 1,4 benzodiazepina, além disso, é rapidamente absorvido e metabolizado no organismo pelo ácido glicurônico e pode causar hipotensão e bradicardia (FARMÁCIA ON-LINE, 2006).

Segundo Schumacher e Yelen (2006), o propofol é o agente anestésico injetável de eleição para a indução de quelônios. É um anestésico geral, do grupo dos barbitúricos, apresentado na fórmula de 2,6-diisopropifenol. Possui pouca solubilidade aquosa, além de ser rapidamente depurado e distribuído pelo organismo. Consequentemente, a indução e recuperação anestésica ocorrem em um curto espaço de tempo. Entre os efeitos colaterais estão queda na pressão arterial, aumento da frequência cardíaca e períodos curtos de apnéia (VALLE et al., 1991).

Este estudo tem como objetivo avaliar os efeitos da associação dos anestésicos midazolan e propofol em cágados tigres d'água brasileiras (Trachemys dorbigni) sobre os parâmetros de manipulação, locomoção, relaxamento muscular, estímulos dolorosos dos membros e cauda, freqüência cardíaca e estimulo oculopalpebral. 
SANTOS, A.L.Q. et al. Anestesia de tigre d'água brasileiro Trachemys dorbigni (DUMÉRIL e BIBRON, 1835) - Testudine: Emydidae com a associação de midazolam e propofol. PUBVET, Londrina, V. 5, N. 17, Ed. 164, Art. 1109, 2011.

\section{MATERIAL E MÉTODOS}

Foram utilizados dez exemplares de Trachemys dorbigni, provenientes de apreensão do IBAMA, no município de Uberlândia (MG), os quais foram mantidos no LAPAS (Laboratório de Ensino e Pesquisa em Animais Silvestres), alojados em tanques com água corrente e temperatura média de $27^{\circ} \mathrm{C}$. Os animais foram pesados e identificados através de um número feito com bisturi cirúrgico na parte inferior do casco.

A administração das drogas foi realizada com seringas descartáveis (BD Plastipak $($ ) ) de $1 \mathrm{ml}$ e $3 \mathrm{ml}$ e agulhas (BD $13 \times 4,5 \mathrm{G}$ ), o midazolan foi aplicado na musculatura dos membros torácicos e o propofol de forma intravenosa. A dosagem utilizada foi de $2 \mathrm{mg} / \mathrm{kg}$ de midazolam como pré anestésico e propofol $10 \mathrm{mg} / \mathrm{kg}$ como anestésico .

Durante o período trans anestésico, os cágados foram avaliados em relação a parâmetros anestésicos nos tempos decorridos de 5, 10, 15 e 20 minutos após a aplicação do midazolam e nos tempos $0,5,10,20,30,45$, $60,90,120,150,180$ minutos após a aplicação do propofol. Os batimentos cardíacos dos animais foram monitorados com o aparelho Doppler Vascular Eletrônico nos tempos $0^{\prime}, 10^{\prime}$, 30', 60', 120' e 180' pós-anestésico.

Os parâmetros estipulados para avaliação anestésica foram: locomoção, relaxamento muscular e estímulos dolorosos nos membros e cauda. Utilizou-se um escore subjetivo de 1 para efeito mínimo, 2 para efeito intermediário e 3 para efeito profundo, para os três primeiros parâmetros. Para os testes de estímulo no membro torácico e cauda, foi utilizada uma pinça hemostática Kelly curva, de $16 \mathrm{~cm}$, com pressionamento até a segunda trava do instrumento, nos espaços interdigitais do membro e cutâneo da pele, respectivamente, e considerado escore 0 para presença de resposta e a ausência, escore 1.

1) Locomoção: capacidade de se locomover normalmente.

2) Relaxamento muscular: capacidade de manter a cabeça elevada, facilidade do avaliador de estender os membros. 
SANTOS, A.L.Q. et al. Anestesia de tigre d'água brasileiro Trachemys dorbigni (DUMÉRIL e BIBRON, 1835) - Testudine: Emydidae com a associação de midazolam e propofol. PUBVET, Londrina, V. 5, N. 17, Ed. 164, Art. 1109, 2011.

3) Manipulação: facilidade do avaliador em manipular manualmente (flexão e extensão) da cabeça, dos membros e da cauda. Facilidade de abrir a boca do animal.

4) Estímulo do membro torácico e pélvico: percepção da dor pelo animal ao pinçamento, com retração do membro ou tentativa de locomoção.

5) Estímulo da cauda: Movimentação da cauda ou tentativa de locomoção do animal após pinçamento da mesma.

6) Freqüência cardíaca: aferição dos batimentos cardíacos através do Doppler vascular.

7) Reflexos protetores oculopalpebrais: retração do globo ocular e fechamento das pálpebras ao toque na córnea.

Os animais foram considerados anestesiados quando o somatório dos escores máximos para locomoção (3), relaxamento muscular (3), manipulação (3), estímulo no membro torácico (1) e estímulo da cauda (1) eram iguais a 11 , e considerados recuperados quando suas atividades se aproximavam às de antes da administração das drogas. A temperatura ambiente permaneceu em torno de 28 á $30^{\circ} \mathrm{C}$ durante o experimento.

A umidade relativa e a temperatura ambiente foram medidas através termohigromêtro, disposto no local do experimento.

Para análise estatística das alterações nos parâmetros causados pela associação dos anestésicos, foi utilizado teste de Friedman, foram considerados estatisticamente significativos resultados menores de 0,05.

\section{RESULTADOS E DISCUSSÃO}

A temperatura média do ambiente permaneceu em torno de 28 a $30^{\circ} \mathrm{C}$ considerando que a temperatura ótima para os répteis para apresentarem melhor funcionamento do seu metabolismo oscila em torno de 28 á $36{ }^{\circ} \mathrm{C}$ (BENNETT, 1991).

O midazolan ultilizado como pré anestésico não provocou bom relaxamento muscular, os animais após 20 minutos da aplicação estavam 
SANTOS, A.L.Q. et al. Anestesia de tigre d'água brasileiro Trachemys dorbigni (DUMÉRIL e BIBRON, 1835) - Testudine: Emydidae com a associação de midazolam e propofol. PUBVET, Londrina, V. 5, N. 17, Ed. 164, Art. 1109, 2011.

ativos e responsivos. Resultado semelhante foi observado por Alves Júnior (2006), ao utilizar a combinação de midazolam e cetamina em Podocnemis expansa; de acordo com o autor, não houve bom relaxamento muscular nos grupos anestesiados com midazolam ( $2 \mathrm{mg} \mathrm{kg-1} \mathrm{IM-1)} \mathrm{cetamina} \mathrm{(20} \mathrm{mg} \mathrm{kg-1}$ IM-1), midazolam (2 mg kg-1 IM-1) e cetamina (60 mg kg-1IM1).

Todos os animais chegaram ao somatório de 11 pontos nos escores, sendo 3 para manipulação, relaxamento muscular e locomoção e 1 para estimulo dolorosos dos membros e cauda. Esse estado permaneceu em média por 23 minutos.

Após 1 minuto da aplicação do anestésico o relaxamento muscular foi observado e se manteve em média por 114 minutos, resultado semelhante foi encontrado por Duke (1995), que considera o relaxamento muscular uma das principais vantagens do propofol, o que permite a execução de procedimentos rápidos.

Os parâmentros de manipulação e locomoção após a aplicação do propofol chegaram ao escore 3 em todos animais, caracterizando uma boa indução anestésica. A manipulação como o observado no relaxamento muscular durou em média 114 minutos e a locomoção por um período maior, em média de 255 minutos.

Em relação à sensibilidade a estímulos dolorosos, a ausência de dor foi em média de 22 minutos para os membros e cauda, após esse período foi observado retorno dos membros pélvicos e cauda, como observado por Bennett (1991) que afirma que os anestésicos em répteis levam a diminuição dos reflexos e do tônus muscular, à medida que a profundidade da anestesia aumenta, progredindo dos membros torácicos para os pelvinos e que durante a recuperação, as funções dos membros retornem em sentido inverso.

Não houve diminuição significativa da frequência cardíacanos animais estudados. Alves Júnior (2006), ao contrário, observou que a dose maior de propofol (10 mg kg-1 IV-1), utilizada no grupo em que foi aplicada xilasina (1,5 mg kg-1 IM-1) e propofol (10 mg kg-1 IV-1), interferiu diretamente para a redução dos batimentos cardíacos das tartarugas-da-amazônia. Fato também 
SANTOS, A.L.Q. et al. Anestesia de tigre d'água brasileiro Trachemys dorbigni (DUMÉRIL e BIBRON, 1835) - Testudine: Emydidae com a associação de midazolam e propofol. PUBVET, Londrina, V. 5, N. 17, Ed. 164, Art. 1109, 2011.

relatado por Magella e Cheibub (1990), ao observarem que, quanto maior a dose de propofol, maior a depressão no aparelho cardiovascular.

De acordo com Ávila Júnior (2005), a aplicação de propofol, em bolos, resultou em alto índice de apneia em todas as Podocnemis expansa estudadas, o que provocou sintomas de cianose da mucosa oral e da língua, apesar desta observação nenhum animal foi a óbito, pois os répteis são capazes de permanecer por tempo prolongado em apneia, podendo fazer respiração anaeróbia (BENNETT, 1996). Este fato foi observado no presente estudo, mesmo com a aplicação do propofol de forma lenta, os animais apresentaram estado de apnéia, porém nenhum animal foi á óbito .

\section{CONCLUSSÃO}

O propofol na dose de $10 \mathrm{mg} / \mathrm{kg}$ foi considerado um bom anestésico levando a um bom relaxamento muscular, analgesia com rápida indução e por tempo prolongado.

O midazolan não se apresentou como um bom pré anestésico para espécie, pois não houve relaxamento muscular após sua aplicação, sendo este o principal parâmetro avaliado em relação a fármacos pré anestésicos .

Porém a associação propofol $(10 \mathrm{mg} / \mathrm{kg})$ e midazolan $(2 \mathrm{mg} / \mathrm{kg})$ foi eficiente, levou á analgesia dos membros e cauda por mais de 20 minutos, podendo ser indicada para pequenos procedimentos cirúrgicos e coletas de materiais para exames laboratoriais.

\section{REFERÊNCIAS}

ALVES JÚNIOR, J. R. F. Anestesia de tartarugas-da-amazônia (Podocnemis expansa) com as associações de cetamina e propofol, midazolam e propofol e midazolam e cetamina. 2006. 43 f. Dissertação (Mestrado)-Faculdade de Medicina Veterinária, Universidade Federal de Uberlândia, Uberlândia, 2006.

AVILA JUNIOR, R. H. Efeitos anestésicos do propofol e do etomidato isolados e associados com butorfanol e fentanila em tartaruga-da amazônia (Podocnemis expansa-Schweigger, 1812). 2005. 31 f. Dissertação (Mestrado)-Faculdade de Medicina Veterinária, Universidade Federal de Uberlândia, Uberlândia. 
BARBOUR, R. W. Turtles of the World, Washington D.C.: Smithsonian Institution Press, 1989, 313p.

BENNETT, R. A. A review of anesthesia and chemical restrain in reptiles. Journal of Zoo and Wildlife Medicine, Philadelphia, v. 22, 1991, p. 282-303.

BENNETT, R. A. Anesthesia. In: MADER, D. R. Reptile medicine and surgery. Philadelphia: W.B. Saunders, 1996, p. 241-247.

CARVALHO, S. F. M. Anestesia dissociativa em Tartarugas da Amazônia (Podocnemis expansa, Schweigger-1812) (Testudinata) e monitoramento cardíaco com

eletrocardiógrafo. 2005. 68 f. Dissertação (Mestrado) - Faculdade de Medicina Veterinária, Universidade Federal de Uberlândia, Uberlândia, 2005.

DARRYL, J. H. Reptile anesthesia. In: Animal care and use. Flórida, 2000. p. 12 T,C.H.;

DUKE, T. A new intravenous anesthetic agent: Propofol. Canadian Veterinary Journal, Ottawa, v.36, p.138 -181, 1995.

FARMÁCIA ON-LINE. Virtualink Network. Disponível em http://www.farmacia.med.br/ medicamentos. Acessado em 02 dez 2010.

FERREIRA YUKI V. L.; DAMBORENEA, M.C.; OSORIO MALLMAN, M. T. Acantochelys spixii (Duméril et Bibron, 1835) (Chelidae) e Trachemys dorbigni (Duméril et Bibron, 1835) (Emydidae) (Testudines) como hospedeiros de Temnocephala brevicornis (Monticelli, 1889) (Temnocephalidae) (Platyhelminthes). Comunicações do Museu de Ciências da PUCRSserie zoologia. v.6, p.75-83, 1993.

HOLZ, P.; BARKER, I. K.; BURGER, J. P.; CONLON, P. D. The effect of the renal portal system on pharmacokinectic parameters in the red eared slider (Trachemis scripta elegans). Journal of Zoo and Wild life Medicine, v.28, p.386-393, 1997.

HOLZ, P.; BARKER, I.K.; CRAWSHAW, G.J.; DOBSON, H. The anatomy and perfusion of the renal portal system in the red eared slider (Trachemis scripta elegans).

Journal of Zoo and Wild life Medicine, v.28, n.4, p.378-385, 1997

SANTANA, E. P. Esofagotomia cervical em tartarugas-da-amazônia (Podocnemis expansa). CD-room. Monografia (graduação) - Faculdade de Medicina Veterinária, Universidade Federal de Uberlândia. Uberlândia, MG, 2003.

SCHUMACHER, J.; YELEN, T. Anesthesia and analgesia. In: MADER, D. R. Reptile medicine and surgery. 2. ed., Missouri: Saunders Elsevier, 2006. p. 442-452.

VALLE, L. B. S.; OLIVEIRA FILHO, R. M.; De LUCIA, R.; OLGA, S. Farmacologia integrada: anestésicos gerais. Rio de Janeiro: Atheneu, 1991. 